\title{
Clinico-radiological evaluation of post COVID-19 at a tertiary pulmonary care centre in Delhi, India
}

\author{
Nitin Goel, Nitesh Goyal, Raj Kumar \\ Department of Pulmonary Medicine, Vallabhbhai Patel Chest Institute, University of Delhi, India
}

\begin{abstract}
In the present COVID-19 pandemic situation, there is a gradual increase in number of patients with post-COVID-19 sequalae. The present study is a retrospective analysis of these post- COVID-19 patients presenting to one of the units of Viswanathan Chest Hospital, Vallabhbhai Patel Chest Institute, University of Delhi, Delhi in the period from 17 June 2020 to 22 October 2020. We analysed the demographic profile, history, functional assessment and all investigations of this study cohort. Out of the 2,165 patients provided consultation, there were 35 patients of post-COVID-19, which were included in the present study. These patients had a mean duration of 47 days from discharge to first visit to our hospital. Preexisting respiratory comorbidity was present in $63 \%$. History of hospitalisation was present in $52 \%$. Fatigue $(65 \%)$ was the most
\end{abstract}

Correspondence: Nitin Goel, M.D. Assistant Professor, Viswanathan Chest Hospital, Department of Pulmonary Medicine, Vallabhbhai Patel Chest Institute, University of Delhi, Room No. 2, Delhi 110007, India.

Tel. +91.9717317899 - +91.011.27667667 Ext: 185 .

E-mail: drnitingoel@gmail.com

Contributions: All the authors made a substantive intellectual contribution, performed part of the experiments. All the authors have read and approved the final version of the manuscript and agreed to be accountable for all aspects of the work.

Conflict of interest: The authors declare that they have no competing interests, and all authors confirm accuracy.

Ethics approval and consent to participate: The present study was approved by the Institutional Ethics Committee (IEC) and informed consent was obtained from the participants.

Key words: COVID-19; lung diseases; comorbidity; pandemic; severe acute respiratory syndrome coronavirus 2 ; tuberculosis.

Received for publication: 17 November 2020.

Accepted for publication: 2 March 2021.

${ }^{\circ}$ Copyright: the Author(s), 2021

Licensee PAGEPress, Italy

Monaldi Archives for Chest Disease 2021; 91:1682

doi: 10.4081/monaldi.2021.1682

This article is distributed under the terms of the Creative Commons Attribution Noncommercial License (by-nc 4.0) which permits any noncommercial use, distribution, and reproduction in any medium, provided the original author(s) and source are credited. common symptom followed by breathlessness (60\%), cough (45.71\%) and chest pain (28.57\%). Three patients had significant desaturation on 6-minute walk test and one patient had type 2 respiratory failure on presentation. Chest X-ray was abnormal in $34.28 \%$ $(n=12)$. On thorax $\mathrm{CT}(\mathrm{n}=17)$ the most common finding was diffuse reticulations $(52.94 \%)$ followed by diffuse ground glass opacities (GGOs) (35.29\%). One patient each were newly diagnosed as pulmonary tuberculosis and tubercular unilateral hilar lymphadenopathy. Conclusively, post-COVID-19 patients may have remnant symptoms like fatigue, breathlessness and cough. Also, patients with pre-existing respiratory diseases are more symptomatic and even may suffer from deterioration in the clinical course. Further we need to be alert of alternate diagnosis or infections like tuberculosis (TB) in these patients, especially in TB endemic countries like India. Simultaneously, the use of immunosuppressant drugs like steroids for COVID-19 management, predisposes to TB. A proper evaluation with holistic and standardised management plan is the need of the hour for post-COVID-19 patients, until its time course, evolution and manifestations are unravelled.

\section{Introduction}

Coronavirus disease 2019 or COVID-19 first emerged in China and soon was declared public health emergency of international concern by World Health Organization on $30^{\text {th }}$ January 2020 [1]. As on $1^{\text {st }}$ November 2020 , globally there have been $45,428,731$ confirmed cases of COVID-19, including 1,185,721 deaths [2]. These figures for India stand as 8,137,119 confirmed cases of COVID-19 with 121,641 deaths [3]. Simultaneously there has been a rise in number of patients who have recovered from COVID-19 disease, in India this figure being 7,491,513 as on $1^{\text {st }}$ November 2020 [4].

Initially, to control the pandemic situation the main target was infection control measures so as to prevent further spread, achieve early diagnosis, supportive and inpatient care for the moderate to severe COVID cases and along with measures for effective treatment and vaccine development. Now as the number of recovered cases of COVID-19 are increasing, there has also been a rise in number of patients with persistent symptoms even after turning negative for severe acute respiratory syndrome coronavirus 2 (SARS-CoV-2). In a study it was found that almost $87.4 \%$ post COVID-19 patients, reported persistence of at least 1 symptom, most common symptoms being fatigue and dyspnoea [5]. Another study found that almost $71 \%$ had radiologic abnormalities and about $25 \%$ had lung function abnormalities even after 3 months of discharge following COVID-19 [6].

In the present study, we evaluated the clinical and radiologic sequalae in post-COVID-19 patients, presenting to a tertiary care centre with various complaints. 


\section{Materials and Methods}

Based on the Ministry of Health and Family Welfare (MOHFW) of the Government of India (GOI) guidelines [7] some government hospitals were designated as COVID hospitals. However, our hospital i.e., Viswanathan Chest Hospital, Vallabhbhai Patel Chest Institute, University of Delhi, was designated as non-COVID hospital and has been catering to all patients of acute/chronic respiratory ailments, other than the COVID-19. Hence, we have also been providing consultation to patients who were diagnosed as COVID-19 and now been discharged from COVID hospital/ home isolation after completion of mandatory isolation as per MOHFW, GOI guidelines [8,9].

The present study is a retrospective analysis of these patients who had previously been diagnosed as COVID-19 (based on a positive RT-PCR for SARS-CoV-2) and presented to one of the units of Viswanathan Chest Hospital, Vallabhbhai Patel Chest Institute, University of Delhi, Delhi in the period from 17 June 2020 to 22 October 2020. We analysed the demographic profile, clinical history, symptoms and signs of this study cohort. Also, the functional assessment, laboratory investigations and radiological investigations were retrieved from the file records, compiled and analysed. Two pulmonologists with more than 14 years and 25 years of clinical experience respectively, analysed all chest X-rays and thorax CT-scans of the study cohort.

\section{Results}

About 2,165 patients were provided consultation in the concerned unit of the department of pulmonary medicine of Viswanathan Chest Hospital, Vallabhbhai Patel Chest Institute between 17 June 2020 to 22 October 2020. Out of these, there were 35 confirmed cases of COVID-19 who had presented after having been discharged from hospital/ home isolation. These postCOVID-19 patients were included in the present study. The various clinical characteristics of the study cohort are depicted in Table 1. The mean duration from discharge (from hospitalisation/home isolation during COVID-19) to first presentation/visit to our hospital was found to be $46 \pm 36$ days. One of the patients was directly shifted from COVID hospital to our set up on completion of the mandatory isolation and testing RT-PCR negative for SARS-CoV2. Out of these 35 post-COVID-19 patients, 10 had pneumonia on chest X-ray when they were diagnosed to have COVID-19. About $49 \%(n=17)$ patients had been managed by home isolation only and $1(2.86 \%)$ patient each had history of non-invasive and invasive mechanical ventilation. Almost $91 \%(n=32)$ patients had one or more comorbidity. Respiratory comorbidity $(62.85 \%)$ was the commonest comorbidity and amongst them bronchial asthma with allergic rhinitis was the most common (14.28\%) diagnosis followed by bronchial asthma $(11.42 \%)$. In the non-respiratory comorbidities, commonest comorbidity was hypertension (22.85\%). On evaluation of post-COVID-19 clinical presentation, fatigue $(65 \%)$ was the most common symptom followed by breathlessness (60\%), cough (45.71\%) and chest pain $(28.57 \%)$. Examination revealed rhonchi in $14 \%$ and crepitations in $11 \%$ of the patients. Three patients had significant desaturation on 6minute walk test and one patient had type 2 respiratory failure on presentation. On laboratory investigations, anaemia was present in about $40 \%$ of patients and thrombocytopenia in about $11 \%$ (Table 2). On radiologic evaluation chest X-ray was abnormal in $34.28 \%$ $(n=12)$ of post-COVID-19 patients and out of these $14.28 \%(n=5)$ patients had diffuse reticulations (Figure 1a). CT thorax was done in patients with resting hypoxemia or desaturation on six-minute walk test (6MWT) or any new opacity/finding from the baseline chest X-ray. Also, if the baseline chest X-ray was not available and the patient had any opacity/ finding on chest X-ray, the CT scan thorax was done. There were in total 17 such patients in whom CT scan was done. All these had abnormal CT scan (Figure $1 \mathrm{~b}$ and Figure 2). Diffuse nodular shadows were present in $5(29.41 \%)$ and diffuse reticulations were present in $9(52.94 \%)$ patients out of these $(n=17)$ (Table 2).

Further an analysis of premorbid respiratory diagnosis of patients with abnormal chest X-ray $(\mathrm{n}=12)$ and abnormal $\mathrm{CT}$ scan thorax $(n=17)$ was done. In the abnormal chest X-ray group, most

Table 1. Characteristics of post-COVID-19 patients $(n=35)$.

\begin{tabular}{|c|c|}
\hline Gender - Male:female & 19:16 \\
\hline Age (mean \pm SD) (in years) & $44.62 \pm 15.77$ \\
\hline Duration to presentation (mean \pm SD) (in days) & $46.37 \pm 36.67$ \\
\hline $\begin{array}{ll}\text { Course/management of COVID-19 } \\
\text { i. } & \text { Home isolation } \\
\text { ii. } & \text { Hospitalisation without oxygen support } \\
\text { iii. } & \text { Hospitalisation with oxygen support } \\
\text { iv. } & \text { Hospitalisation non-invasive ventilation (NIV) } \\
\text { v. } & \text { Hospitalisation invasive mechanical ventilation (IMV) }\end{array}$ & $\begin{array}{l}n(\%) \\
17(48.57) \\
11(31.43) \\
5(14.29) \\
1(2.86) \\
1(2.86)\end{array}$ \\
\hline 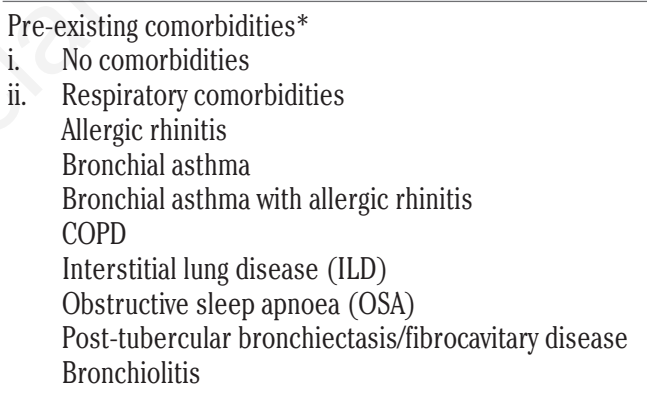 & $\begin{array}{l}3(8.57) \\
22(62.85) \\
2(5.71) \\
4(11.42) \\
5(14.28) \\
3(8.57) \\
1(2.85) \\
1(2.85) \\
5(14.28) \\
1(2.85)\end{array}$ \\
\hline $\begin{array}{l}\text { iii. Non respiratory comorbidities } \\
\text { Hypertension } \\
\text { Diabetes mellitus } \\
\text { Coronary artery disease and dyslipidaemia } \\
\text { Hypothyroidism } \\
\text { Hyperthyroidism } \\
\text { Psychiatric diseases } \\
\text { Epilepsy }\end{array}$ & $\begin{array}{l}18(51.42) \\
8(22.85) \\
6(17.14) \\
2(5.71) \\
2(5.71) \\
3(8.57) \\
3(8.57) \\
1(2.85)\end{array}$ \\
\hline Post-COVID-19 presentation: symptom/sign & \\
\hline i. Cough & $16(45.71)$ \\
\hline ii. Sputum & $4(11.43)$ \\
\hline iii. Fatigue & $23(65.71)$ \\
\hline iv. Breathlessness & $21(60)$ \\
\hline v. Wheezing & $6(17.14)$ \\
\hline vi. Chest pain & $10(28.57)$ \\
\hline vii. Fever & $3(8.57)$ \\
\hline viii. Haemoptysis & $0(0)$ \\
\hline ix. Loss of appetite & $4(11.43)$ \\
\hline x. Loss of weight & $2(5.71)$ \\
\hline xi. Tachycardia & $4(11.43)$ \\
\hline xii. Raised blood pressure & $8(22.86)$ \\
\hline xiii. Tachypnoea & $1(2.86)$ \\
\hline xiv. Resting $\mathrm{SpO}_{2}<95 \%$ & $1(2.86)$ \\
\hline xv. Rhonchi on auscultation & $5(14.29)$ \\
\hline xvi. Crepitations on auscultation & $4(11.43)$ \\
\hline
\end{tabular}


common diagnosis was post-COVID-19 with no pre-existing respiratory disease $(n=7,55.11 \%)$ followed by bronchial asthma with allergic rhinitis $(n=2,16.66 \%)$. On analysis of the patients with abnormal CT thorax, maximum patients had a diagnosis of postCOVID-19 with no pre-existing respiratory disease $(n=8,47.05 \%)$ followed by bronchial asthma with allergic rhinitis $(n=3,17.64 \%)$. One patient each were newly diagnosed as pulmonary tuberculosis and tubercular unilateral hilar lymphadenopathy

\section{Discussion}

Ever since the beginning of the COVID-19 pandemic, there has been rapidly increasing number of affected populations worldwide. Hence, the main emphasis of the health care systems has been on the preventive and therapeutic aspects of the COVID-19.

Table 2. Investigations of post COVID-19 patients.

\begin{tabular}{|c|c|}
\hline Investigations & n $(\%)$ \\
\hline Desaturation in six-minute-walk-test & $3(8.57)$ \\
\hline Type 2 respiratory failure on $\mathrm{ABG}$ & $1(2.86)$ \\
\hline Anaemia $(\mathrm{Hb}<13.5 \mathrm{gm} / \mathrm{dL}$ in males; $\mathrm{Hb}<12.5 \mathrm{gm} / \mathrm{dL}$ in females) & $14(40)$ \\
\hline $\begin{array}{l}\text { Leukocytopenia }\left(\mathrm{TLC}<4000 \text { cells } / \mathrm{mm}^{3} \text { ) }\right. \\
\text { Leucocytosis }\left(\mathrm{TLC}>10,000 \text { cells } / \mathrm{mm}^{3} \text { ) }\right.\end{array}$ & $\begin{array}{l}3(8.57) \\
7(20)\end{array}$ \\
\hline $\begin{array}{l}\text { Neutropenia }(\mathrm{N}<40 \%) \\
\text { Neutrophilia }(\mathrm{N}>80 \%)\end{array}$ & $\begin{array}{l}1(2.86) \\
1(2.86)\end{array}$ \\
\hline $\begin{array}{l}\text { Lymphopenia }(\mathrm{L}<20 \%) \\
\text { Lymphocytosis }(\mathrm{L}>40 \%)\end{array}$ & $\begin{array}{l}4(11.43) \\
1(2.86)\end{array}$ \\
\hline Monocytosis $(\mathrm{M}>10 \%)$ & $1(2.86)$ \\
\hline Eosinophilia $(\mathrm{E}>6 \%)$ & $2(5.71)$ \\
\hline $\begin{array}{l}\text { Thrombocytopenia (platelets }<150 \times 10^{3} / \mathrm{uL} \text { ) } \\
\text { Thrombocytosis (platelets }>400 \times 10^{3} / \mathrm{uL} \text { ) }\end{array}$ & $\begin{array}{l}4(11.43) \\
3(8.57)\end{array}$ \\
\hline Increased ALT/SGPT (>40 U/L) & $6(17.14)$ \\
\hline Increased AST/SGOT ( >34 U/L) & $9(25.71)$ \\
\hline Increased ALP (>150 U/L) & $26(74.28)$ \\
\hline Increased blood sugars (RBS $>200 \mathrm{mg} / \mathrm{dL}$ or HbAlc $>5.7 \%$ ) & $5(14.28)$ \\
\hline \multicolumn{2}{|l|}{ Chest X-ray finding } \\
\hline Normal study & $23(65.71)$ \\
\hline Diffuse reticulations & $5(14.28)$ \\
\hline Bilateral lower zone reticulations & $4(11.42)$ \\
\hline Cystic changes & $2(5.71)$ \\
\hline Unilateral hilar lymphadenopathy & $1(2.85)$ \\
\hline \multicolumn{2}{|l|}{ CT-thorax finding ( $\mathrm{n}=17)$} \\
\hline Normal study & $0(0)$ \\
\hline Diffuse nodular shadows & $5(29.41)$ \\
\hline Diffuse reticulations & $9(52.94)$ \\
\hline Bilateral lower lobe reticulations & $1(5.88)$ \\
\hline Diffuse ground glass opacities (GGOs) & $6(35.29)$ \\
\hline Bilateral lower lobe GGOs & $2(11.76)$ \\
\hline Bronchiectasis & $1(5.88)$ \\
\hline Fibro-cavitary changes & $1(5.88)$ \\
\hline Emphysematous changes & $2(11.76)$ \\
\hline Unilateral hilar lymphadenopathy & $1(5.88)$ \\
\hline
\end{tabular}

Simultaneously, the numbers of recovered patients from COVID19 are also increasing. In the previous epidemics caused by the coronaviruses, i.e., severe acute respiratory syndrome (SARS) in 2002 and Middle East respiratory syndrome (MERS) in 2012, it was observed that recovered patients had residual lung damage and symptoms. Studies have observed reduced lung function and exercise capacity in almost one-fourth of the survivors of SARS and MERS with history of hospitalisation, even 6 months after discharge [10]. Even after one year post discharge these patients were found to suffer from psychologic disturbances like depression, anxiety and post- traumatic stress disorder along with poor quality of life. Based on these, the residual changes / sequalae in post COVID-19 survivors are expected to be likewise.

COVID-19 caused by SARS-CoV-2 coronavirus has a variable symptomatology and clinical course in different individuals ranging from asymptomatic, mild symptoms to even severe disease manifesting acute respiratory distress syndrome (ARDS) and multi-organ dysfunction syndrome [11]. Initial findings from the COVID-19 pandemic suggest that long term respiratory abnormalities in the form of post COVID-19, may occur in some of the survivors. Studies have found fibrotic changes in the lung irrespective of the initial severity of COVID-19 [12-14]. In the present study we included 35 patients of post-COVID-19 and analysed their clinical characteristics, symptomatology, laboratory findings and radiology.

We found the mean age of the study patients to be about 44 years. The average duration of presentation of patients for consultation after discharge from the hospitalisation/home isolation was found to be about 6 weeks. One patient had been directly shifted from COVID hospital and the maximum duration of the presentation observed was 124 days. Similar to this observation, studies have found lung function abnormalities and various symptoms even 3 months after discharge from COVID-19 [5]. About 48.5\% of the patients in the present study cohort, recovered in home isolation only whereas about $15 \%$ required oxygen support and one patient each required non-invasive and invasive ventilation respectively (Table 1). Hence, almost $52 \%$ of the present study population required hospitalisation. This may be because of high prevalence of comorbidities (about 91\%) in the study cohort. Since our hospital is a dedicated tertiary pulmonary care centre, majority of the postCOVID-19 patients presenting to us were found to have respiratory comorbidity (63\%). The most common respiratory ailment was bronchial asthma (with or without allergic rhinitis) being about $26 \%$, followed by post- tubercular bronchiectasis/fibrocavitary disease (14\%). Out of the $9(26 \%)$ asthma patients, 4 patients had history of hospital admission (without oxygen support) and 5 patients underwent home isolation during COVID-19. Only 2 asthma patients with history of home isolation had mild asthma and rest all had moderate asthma. A few studies have found lower occurrence of COVID-19 in asthma and a lower number of asthma exacerbations [15]. However, in the present study, asthma was the commonest respiratory comorbidity. This can be due to various confounders like exposure/contact differences and regional variations.

Hypertension (23\%) followed by diabetes mellitus (17\%) was the most common non- respiratory comorbidity. As previously also observed, comorbidities lead to increase in severity of COVID-19 [16] and almost 50\% patients may have oxygen requirement in such type of study population [17]. Further the patients having suffered more severe COVID-19 requiring acute hospital care are more prone and require more aggressive medical care [12]. This was also observed in our study population of post-COVID-19 presenting at our pulmonary set up with $52 \%$ having history of hospitalisation.

The most common symptom in the present post-COVID-19 


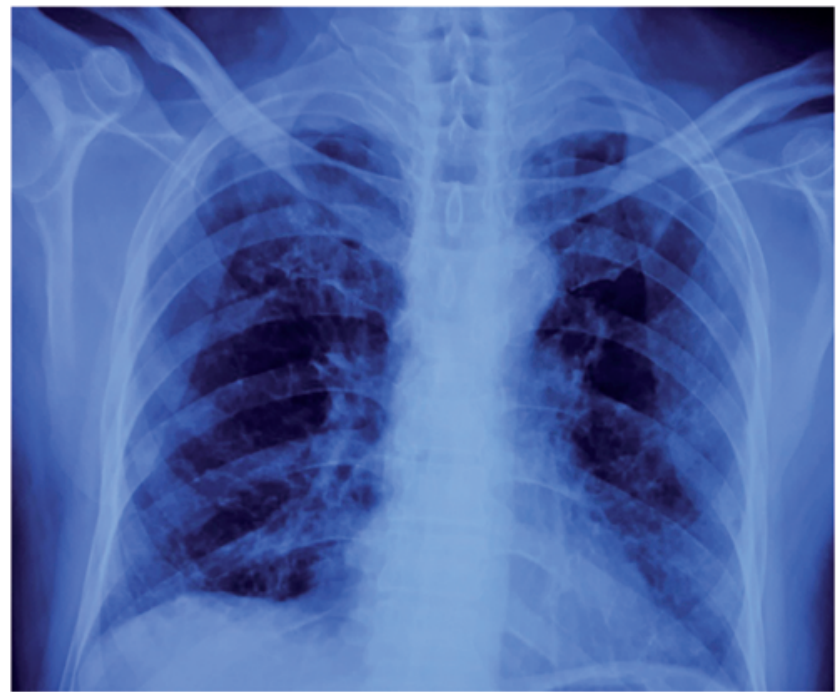

1a

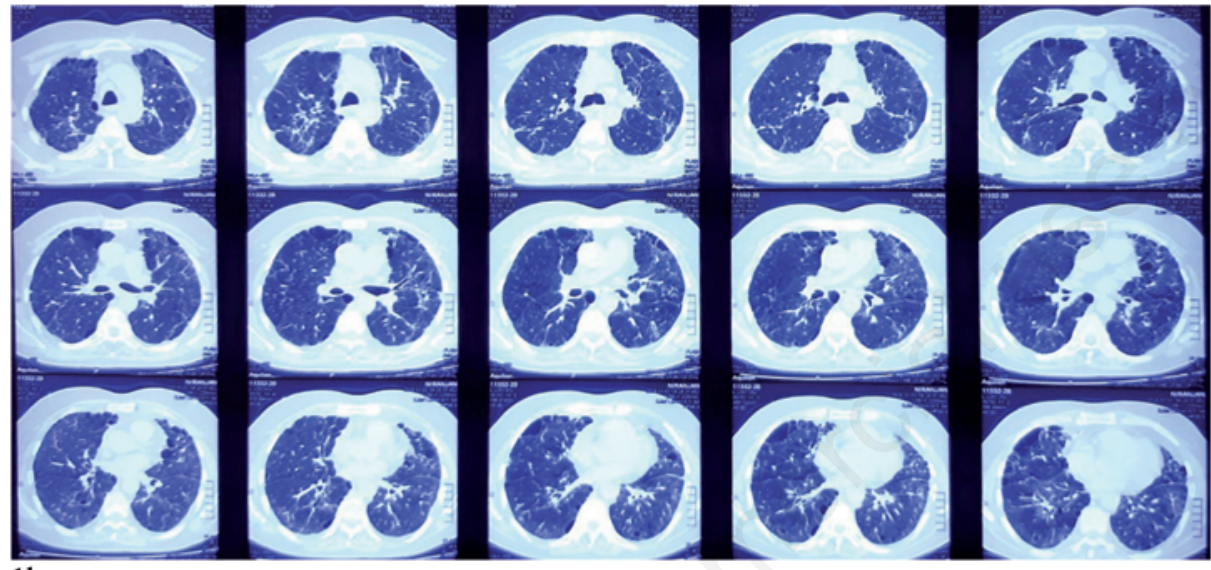

Figure 1. a) Chest X-ray of 68-yearold male COPD with post-COVID19 patient showing bilateral reticulations. b) Thorax CT scan of same patient showing emphysematous changes with areas of ground glass opacities and reticulations.

$1 \mathrm{~b}$

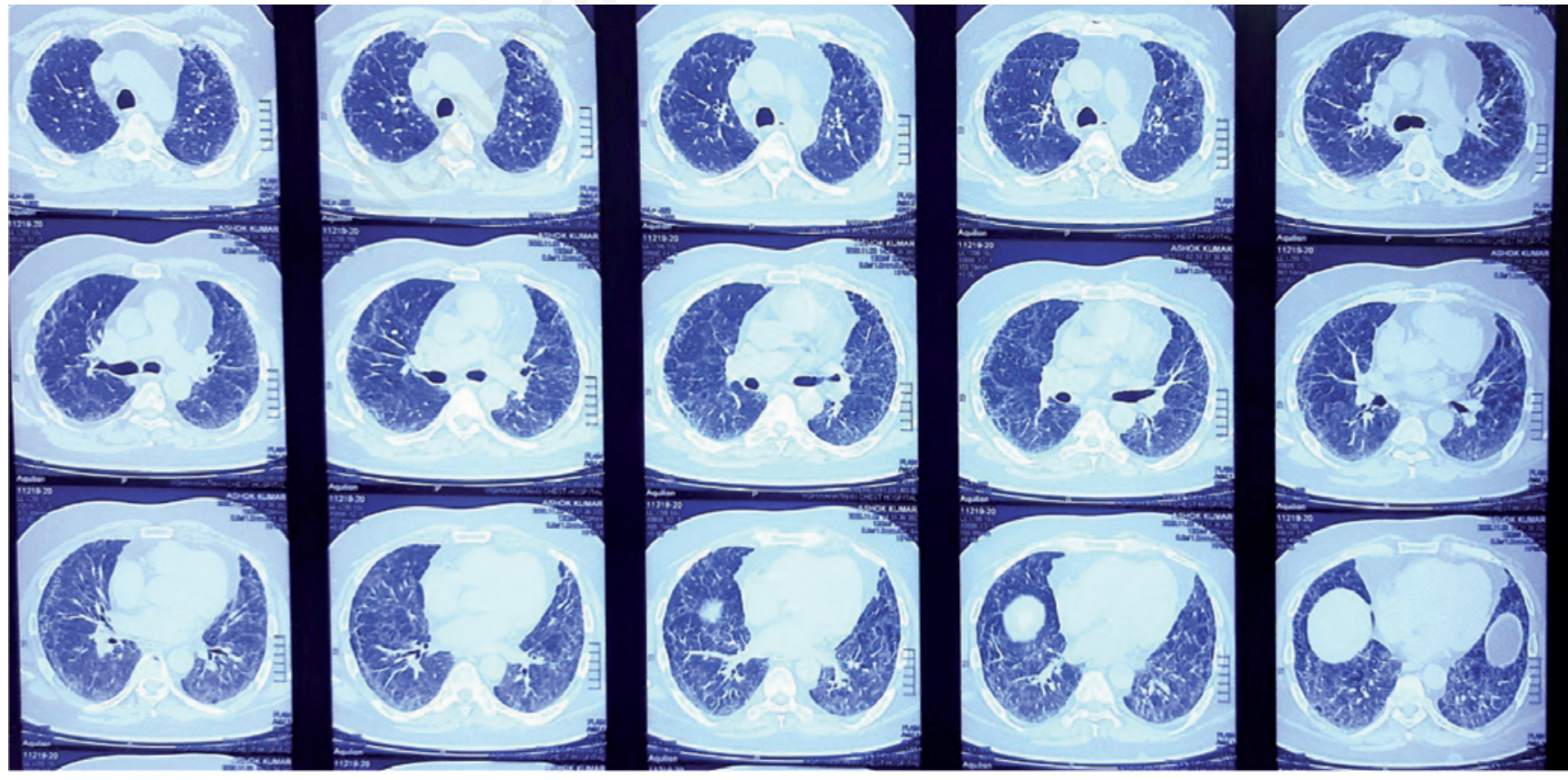

Figure 2. CT scan thorax of 59-year-old male post-COVID-19 patient with no pre-existing respiratory comorbidity showing bilateral ground glass opacities. 
patient population was fatigue $(65 \%)$ followed by breathlessness $(60 \%)$ and cough (45\%). In a study on post COVID-19 patients, it was found that almost $74 \%$ had at least one symptom at $8-12$ weeks post discharge and the most common symptoms reported were breathlessness and fatigue [18]. Another study reported presence of at least one symptom in $87 \%$ of the post-COVID-19 patients [5]. Our findings are similar to these post-COVID-19 studies. Since our patient population were from OPD/emergency department of a pulmonary institute, all were expectedly symptomatic and also respiratory symptoms were the main presenting complaints.

Only one patient had resting hypoxemia on pulse oximetry and he also had type 2 respiratory failure. This patient was a diagnosed follow up case of respiratory bronchiolitis and had developed respiratory failure post-COVID-19. This case scenario emphasizes the significance of respiratory follow up of patients with respiratory comorbidity which can worsen and have a downhill course postCOVID-19. Further three (8.5\%) patients had desaturation on 6minute-walk-test (6MWT).

On chest X-ray analysis, 12 (34\%) patients had abnormal chest $\mathrm{X}$-ray with most common finding being diffuse reticulations in all zones (14\%) followed by bilateral lower zone reticulations (11\%). CT scan thorax was done in patients $(n=17)$ having either one of the following i) resting hypoxemia; ii) if desaturation was present on 6MWT; or iii) abnormal chest X-ray compared to baseline chest $\mathrm{X}$-ray, if available. If the baseline chest X-ray was not available and the patient had any opacity/ finding on chest X-ray, then also thorax CT scan was done. All these patients had an abnormal CT scan (Table 2) and diffuse reticulations (53\%) was commonest observation followed by diffuse ground glass opacities (GGOs) $(35 \%)$ and diffuse nodular shadows $(29 \%)$. In a study on thoraxCT of COVID-19 patients done about 24 days from symptom onset, it was found that almost $94 \%$ patients had abnormal CT scans and commonest finding was GGOs [19]. Since CT scans were done in patients on fulfilling the criteria as above, all patients were found to have abnormal CT scans in the present study.

Further, an analysis of respiratory diagnosis in patients with abnormal chest X-ray $(n=12)$ and abnormal CT scan thorax $(n=17)$, was done. On chest X-ray, 55\% $(n=7)$ patients had a diagnosis of post-COVID-19 alone with no pre-existing respiratory disease, followed by bronchial asthma with allergic rhinitis $(17 \%, n=2)$. Analysis of diagnosis of patients who underwent CT scan thorax revealed post-COVID-19 with no pre-existing respiratory disease to be the commonest diagnosis $(47 \%, n=8)$ followed by bronchial asthma with allergic rhinitis $(17 \%, n=3)$. Presence of such abnormalities on CT scan thorax, especially in patients with no preexisting respiratory disease, points to the remnant lung parenchymal damage in post- COVID-19 patients. These would be the causative reason for the various respiratory symptoms/abnormalities in these patients. Further large-scale longitudinal studies are the need of the hour to elucidate the nature, extent and course of these post-COVID-19 pulmonary abnormalities.

Additionally, one patient each were newly diagnosed as pulmonary tuberculosis (TB) and tubercular unilateral hilar lymphadenopathy. The pulmonary tuberculosis patient was diagnosed based on positive sputum smear for acid fast bacilli (AFB). Whereas the patient with unilateral hilar lymphadenopathy had history of anorexia, weight loss and positive Mantoux test. This patient did not consent for bronchoscopy and showed clinical as well radiologic response to antitubercular therapy. Both these patients had history of steroid administration during the course of COVID-19 illness. This again emphasizes the need for proper evaluation of these post-COVID-19 patients for alternate diagnosis/superimposed infections especially tuberculosis. This fact can- not be overemphasized in tuberculosis endemic countries like India and also in the wake of various immunosuppressant drugs like steroids being tried for treatment of COVID-19. Also, link between COVID-19 and active TB disease is not conclusively confirmed $[20,21]$. Whether COVID infection predisposes to development of active TB disease from latent tuberculosis infection or it simply leads presentation of the TB disease at an earlier stage, needs evaluation in large scale studies.

The present study had certain limitations. Firstly, this study is a retrospective evaluation from the self-approached patient visits at a tertiary pulmonary care centre. Hence, there might be an overestimation of symptom burden in the post COVID patients. Second, for the same reason, respiratory comorbidity was the commonest comorbidity present. Further, we had only a single time-point follow up in the present study. Previous studies have suggested (especially in SARS and MERS affected patients) that on serial follow up imaging or pulmonary function tests over 1 year, there may be further spontaneous improvement in the lung function and radiology in these patients $[10,22]$. Hence, the single time point followup in the present study done at around 6 weeks post discharge, may have overestimated the sequelae in the post-COVID-19.

\section{Conclusions}

Post-COVID-19 patients present with various symptoms like fatigue, breathlessness and cough requiring appropriate evaluation and management. Further patients with pre-existing respiratory diseases can manifest more persistent and severe symptoms or even deterioration in clinical course. Hence, emphasis is required to be given for vigilant follow up and management of such patients. Also, we need to be alert of alternate diagnosis or infections like tuberculosis in these patients which otherwise may get missed in the garb of post-COVID-19 symptoms. Lastly, a proper evaluation, follow up and holistic management plan requires to be standardised and practised till we come to know the exact types and time course of post- COVID-19.

\section{References}

1. World Health Organization. Statement on the second meeting of the International Health Regulations (2005) Emergency Committee regarding the outbreak of novel coronavirus (2019nCoV). Accessed: 2020 Nov 1. Available from: https://www.who.int/news/item/30-01-2020-statement-on-thesecond-meeting-of-the-international-health-regulations(2005)-emergency-committee-regarding-the-outbreak-ofnovel-coronavirus-(2019-ncov)

2. World Health Organization. WHO Coronavirus Disease (COVID-19) Dashboard. Accessed: 2020 Nov 1. Available from: https://covid19.who.int

3. World Health Organization. WHO Coronavirus Disease (COVID-19) Dashboard - India. Accessed: 2020 Nov 1. Available from: https://covid19.who.int

4. Ministry of Health and Family Welfare, Government of India [Internet]. COVID-19 Statewise Status. Accessed: 2020 Oct 31. Available from: https://www.mohfw.gov.in/

5. Carfî A, Bernabei R, Landi F, Gemelli against COVID-19 Post-Acute Care Study Group. Persistent symptoms in patients after acute COVID-19. JAMA 2020;324:603-5. 
6. Zhao YM, Shang YM, Song WB, et al. Follow-up study of the pulmonary function and related physiological characteristics of COVID-19 survivors three months after recovery. EClinicalMedicine 2020;25:100463.

7. Ministry of Health and Family Welfare, Government of India [Internet]. Final Guidance on Management of Covid cases version 2. Accessed: 2020 Oct 31. Available from: https://www.mohfw.gov.in/pdf/FinalGuidanceonMangaement ofCovidcasesversion2.pdf

8. Ministry of Health and Family Welfare, Government of India [Internet]. Revised Home Isolation Guidelines. Accessed: 2020 Oct 31. Available from: https://www.mohfw.gov.in/pdf/ RevisedHomeIsolationGuidelines.pdf

9. Ministry of Health and Family Welfare, Government of India [Internet]. Revised discharge policy for COVID19. Accessed: 2020 Oct 31. Available from: https://www.mohfw.gov.in/pdf/ ReviseddischargePolicyforCOVID19.pdf

10. Ahmed H, Patel K, Greenwood DC, et al. Long-term clinical outcomes in survivors of severe acute respiratory syndrome and Middle East respiratory syndrome coronavirus outbreaks after hospitalisation or ICU admission: A systematic review and meta-analysis. J Rehabil Med 2020;52:jrm00063.

11. Wu Z, McGoogan JM. Characteristics of and important lessons from the coronavirus disease 2019 (COVID-19) outbreak in China: Summary of a report of 72314 cases from the Chinese Center for Disease Control and Prevention. JAMA 2020;323: 1239-42.

12. British Thoracic Society. Guidance on respiratory follow up of patients with a clinico- radiological diagnosis of COVID-19 pneumonia. British Thoracic Society, May 11, 2020. Available from: https://www.brit-thoracic.org.uk/document-library/quality-improvement/covid-19/resp-follow-up-guidance-postcovid-pneumonia/
13. Li X, Zeng W, Li X, et al. CT imaging changes of corona virus disease 2019 (COVID- 19): a multi-center study in Southwest China. J Transl Med 2020;18:154.

14. Ahmed H, Patel K, Greenwood D, et al. Long-term clinical outcomes in survivors of coronavirus outbreaks after hospitalisation or ICU admission: a systematic review and metaanalysis of follow-up studies. medRxiv 2020.04.16.20067975.

15. Pignatti P, Visca D, Cherubino F, et al. Impact of COVID-19 on patients with asthma. Int J Tuberc Lung Dis 2020;24:1217-9.

16. Kamal M, Abo Omirah M, Hussein A, Saeed H. Assessment and characterisation of post-COVID-19 manifestations. Int J Clin Pract 2020;e13746. doi:10.1111/ijcp.13746.

17. Goel N, Spalgais S, Mrigpuri P, et al. Characteristics of COVID-19 at a non-COVID tertiary pulmonary care centre in Delhi, India. Monaldi Arch Chest Dis 2020;90:1568.

18. Arnold DT, Hamilton FW, Milne A, et al. Patient outcomes after hospitalisation with COVID-19 and implications for follow-up; results from a prospective UK cohort. medRxiv 2020.08.12.20173526.

19. Wang Y, Dong $\mathrm{C}, \mathrm{Hu} \mathrm{Y}$, et al. Temporal Changes of CT Findings in 90 Patients with COVID-19 Pneumonia: A Longitudinal Study. Radiology. 2020;296:E55-E64. doi:10.1148/ radiol.2020200843.

20. Tadolini M, Codecasa LR, García-García JM, et al. Active tuberculosis, sequelae and COVID-19 co-infection: first cohort of 49 cases. Eur Respir J 2020;56:2001398.

21. Motta I, Centis R, D'Ambrosio L, et al. Tuberculosis, COVID19 and migrants: Preliminary analysis of deaths occurring in 69 patients from two cohorts. Pulmonology 2020;26:233-40.

22. Wong KT, Antonio GE, Hui DS, et al. Severe acute respiratory syndrome: thin-section computed tomography features, temporal changes, and clinicoradiologic correlation during the convalescent period. J Comput Assist Tomogr 2004;28:790-5. 